\title{
Family Genomoviridae: 2021 taxonomy update
}

\author{
Arvind Varsani $^{1,2} \cdot$ Mart Krupovic $^{3}$
}

Published online: 31 July 2021

(C) The Author(s), under exclusive licence to Springer-Verlag GmbH Austria, part of Springer Nature 2021

\begin{abstract}
The family Genomoviridae (phylum Cressdnaviricota, class Repensiviricetes, order Geplafuvirales) includes viruses with circular single-stranded DNA genomes encoding two proteins, the capsid protein and the rolling-circle replication initiation protein. The genomes of the vast majority of members in this family have been sequenced directly from diverse environmental or animal- and plant-associated samples, but two genomoviruses have been identified infecting fungi. Since the last taxonomic update of the Genomoviridae, a number of new members of this family have been sequenced. Here, we report on the most recent taxonomic update, including the creation of one new genus, Gemytripvirus, and classification of $\sim 420$ new genomoviruses into 164 new species. We also announce the adoption of the "Genus + freeform epithet" binomial system for the naming of all 236 officially recognized species in the family Genomoviridae. The updated taxonomy presented in this article has been accepted by the International Committee on Taxonomy of Viruses (ICTV).
\end{abstract}

The family Genomoviridae includes viruses with small circular single-stranded (ss) DNA genomes $(\sim 1.8-2.4 \mathrm{~kb})$ encoding a rolling-circle replication initiation protein (Rep) and a capsid protein $(\mathrm{CP})$ in an ambisense orientation [35]. Whereas the genomoviral $\mathrm{CP}$ is not recognizably similar at the sequence level to the CPs of other known viruses, the Rep is homologous to those of other eukaryotic ssDNA viruses and is most similar to those of plant viruses of the family Geminiviridae, sharing several unique sequence motifs and forming a sister group in phylogenetic analyses [21-23]. Accordingly, the families Genomoviridae and Geminiviridae were included in the order Geplafuvirales [36]. All eukaryotic ssDNA viruses encoding these related Reps, informally referred to as the CRESS DNA viruses [57,

Handling Editor: Sead Sabanadzovic.

Arvind Varsani

arvind.varsani@asu.edu

$\triangle$ Mart Krupovic

mart.krupovic@pasteur.fr

1 The Biodesign Center for Fundamental and Applied Microbiomics, Center for Evolution and Medicine, School of Life Sciences, Arizona State University, Tempe, Arizona, USA

2 Structural Biology Research Unit, Department of Integrative Biomedical Sciences, University of Cape Town, Cape Town, South Africa

3 Archaeal Virology Unit, Institut Pasteur, Paris, France
82], were recently officially unified in the phylum Cressdnaviricota [36, 72].

The founding member of the Genomoviridae [35], Sclerotinia sclerotiorum hypovirulence-associated DNA virus 1 (SsHADV-1), infects the phytopathogenic fungus Sclerotinia sclerotiorum [79] but can also replicate in its transmission vector, the mycophagous insect Lycoriella ingenua [43]. However, the vast majority of genomoviruses have been discovered by metagenomics in diverse samples (see Supplementary Table S1), and the real extent of their host range remains unknown. In 2017, a sequence-based taxonomic framework was established for genomovirus classification [70]. In particular, 78\% genome-wide pairwise identity was chosen as a species demarcation threshold, whereas Rep sequence phylogeny was used to define genera. At the time, the family consisted of 121 members, which were classified based on genome sequences into 73 species divided into nine genera: Gemycircularvirus (43 species and 73 members), Gemyduguivirus (1 species and 1 member), Gemygorvirus (5 species and 9 members), Gemykibivirus (16 species and 29 members), Gemykolovirus (2 species and 3 members), Gemykrogvirus (3 species and 3 members), Gemykroznavirus (1 species and 1 member), Gemytondvirus (1 species and 1 member), and Gemyvongvirus (1 species and 1 member).

In the period since the establishment of the family Genomoviridae [35] and the first report on the classification of then known genomoviruses $(\mathrm{n}=122)$ [70], 420 new complete genome sequences of genomoviruses had 
been deposited in the GenBank database as of May 2020, including both virus isolates and viruses discovered by metagenomics $[2,3,6-9,11-16,18,24,26-34,37-42$, 44-46, 48, 50-56, 58, 59, 61, 62, 64-69, 71, 73-81, 83]. Furthermore, the International Committee on Taxonomy of Viruses (ICTV) has recently adopted a freeform binomial species nomenclature, whereby virus species names have to consist of two words, with the first one being the genus name and the second being a free-form species epithet, which can consist of Latin letters and/or Arabic numerals [60]. All existing species that currently do not conform to this binomial format have to be renamed before 2023 . Here, we report on the classification of the new genomoviruses as well as on other taxonomic changes in the family Genomoviridae, which were approved by the ICTV following the annual ratification vote in March of 2021.

The first notable change implemented in the Genomoviridae taxonomy was adoption of the binomial species nomenclature for all 73 existing species. The correspondence between the old and new binomial species names is shown in Table 1. We note that the names of viruses that are included in the corresponding species are not affected. For instance, the virus name Sclerotinia sclerotiorum hypovirulence associated DNA virus 1 remains unchanged although the species name Sclerotinia gemycircularvirus 1 has been changed to Gemycircularvirus sclerol.

The second taxonomic development involves the classification of $\sim 420$ new genomoviruses. We used the criteria of Rep amino-acid-based phylogeny (Fig. 1) to assign genomoviruses to genera, as outlined by Varsani and Krupovic [70]. By contrast, the recently isolated Fusarium graminearum gemytripvirus 1 (FgGMTV1), infecting Fusarium graminearum, a fungal plant pathogen with worldwide distribution that causes Fusarium head blight (FHB) disease in wheat and barley [39], formed a separate branch in the Rep phylogeny (Fig. 1). Notably, unlike other members of the Genomoviridae, which are monopartite, FgGMTV1 contains three genomic segments, each encoding a single protein [39]: DNA-A encodes a Rep protein; DNA-B encodes a genomovirus-like CP; and DNA-C encodes a protein of unknown function. DNA-A and DNA-B are mutually interdependent for their replication, whereas DNA-C relies on DNA-A and DNA-B for replication and appears to enhance virus pathogenesis and transmission via conidia as well as accumulation of viral DNA in infected fungi [39]. Phylogenetic analysis suggests that the multipartite genome of FgGMTV1 has evolved from a monopartite genome of an ancestral genomovirus. Thus, based on the Rep phylogeny and its multipartite genome organization, FgGMTV1 has been classified as a member of a new species, Gemytripvirus fugral, within a new genus, Gemytripvirus (gemini-like myco-infecting tripartite virus) [39].

Using the previously established species demarcation criteria [70], namely, genome-wide pairwise identity of $78 \%, 35$ viruses can be assigned to eight known species, while the remaining 389 viruses are classified to 164 new species (Table 1). The new species were named using the free-form binomial system. The greatly expanded dataset of genomoviruses has reinforced the validity of the previously established species demarcation criteria. Indeed, pairwise comparison of the representative sequences from each of the species (except for Gemytripvirus fugral, whose members have a tripartite genome) showed that they share less than $78 \%$ genome-wide pairwise identity (Figs. 2 and 3). A summary of the genome sequences of genomoviruses belonging to specific genera and their source are summarized in Figure 4, and additional details are provided in Supplementary Table S1. Accordingly, this threshold will continue to be used for further taxonomic classification of new genomoviruses.

Finally, we note that following the taxonomic assessment described herein, a number of new genomoviruses $(n=201$; GenBank, download 15 May 2021) have been discovered [4, $5,10,25,63]$. Of these, 42 can be assigned to currently established species, and 161, once classified, are likely to represent new species and genera within the family Genomoviridae. However, we would like to discourage naming newly discovered viruses using official taxon names. For instance, an ssDNA virus infecting the phytopathogenic fungus Botrytis cinerea has been isolated recently and named Botrytis cinerea genomovirus 1 (BcGV1) [17]. Although BcGV1 displays a genomic organization similar to that of genomoviruses and encodes a related Rep, the CP encoded by this virus is unrelated to that of genomoviruses. Thus, placement of BcGV1 within the family Genomoviridae is questionable. 
Table 1 Nomenclatural and taxonomic changes in the family Genomoviridae

\begin{tabular}{|c|c|c|c|c|}
\hline Genus & New species name & Former species name & Exemplar virus & Accession no. \\
\hline \multirow[t]{31}{*}{ Gemycircularvirus } & Gemycircularvirus abatil & - & Genomoviridae sp. & MK032723 \\
\hline & Gemycircularvirus alces 1 & - & $\begin{array}{l}\text { Alces alces faeces associated } \\
\text { genomovirus MP84 }\end{array}$ & MG641204 \\
\hline & Gemycircularvirus alces2 & - & $\begin{array}{l}\text { Alces alces faeces associated } \\
\text { genomovirus MP157 }\end{array}$ & MG641207 \\
\hline & Gemycircularvirus ansall & - & Genomoviridae sp. & MN928911 \\
\hline & Gemycircularvirus asparl & - & Plant associated genomovirus 21 & MH939436 \\
\hline & Gemycircularvirus austrol & - & Blackfly genomovirus 2 & MK433234 \\
\hline & Gemycircularvirus austro2 & - & Blackfly genomovirus 7 & MK433239 \\
\hline & Gemycircularvirus austro3 & - & Blackfly genomovirus 4 & MK433236 \\
\hline & Gemycircularvirus austro4 & - & Blackfly genomovirus 9 & MK433241 \\
\hline & Gemycircularvirus austro5 & - & Blackfly genomovirus 5 & MK433237 \\
\hline & Gemycircularvirus austro6 & - & Blackfly genomovirus 10 & MK433242 \\
\hline & Gemycircularvirus bemtal & - & $\begin{array}{l}\text { Bemisia-associated genomovirus } \\
\text { AdO }\end{array}$ & KY230614 \\
\hline & Gemycircularvirus blabil & $\begin{array}{l}\text { Blackbird associated gemycircular- } \\
\text { virus } 1\end{array}$ & $\begin{array}{l}\text { Faecal-associated gemycircularvirus } \\
\text { 1a }\end{array}$ & KF371643 \\
\hline & Gemycircularvirus bovas 1 & $\begin{array}{l}\text { Bovine associated gemycircularvi- } \\
\text { rus } 1\end{array}$ & $\begin{array}{l}\text { Faeces associated gemycircularvirus } \\
22\end{array}$ & KT862253 \\
\hline & Gemycircularvirus bromas 1 & $\begin{array}{l}\text { Bromus associated gemycircular- } \\
\text { virus } 1\end{array}$ & $\begin{array}{l}\text { Bromus-associated circular DNA } \\
\text { virus } 3\end{array}$ & KM510192 \\
\hline & Gemycircularvirus canlup1 & - & $\begin{array}{l}\text { Lupine feces-associated gemycircu- } \\
\text { larvirus } 2\end{array}$ & KY214442 \\
\hline & Gemycircularvirus cassal & $\begin{array}{l}\text { Cassava associated gemycircular- } \\
\text { virus } 1\end{array}$ & $\begin{array}{l}\text { Cassava associated cicular DNA } \\
\text { virus }\end{array}$ & JQ412056 \\
\hline & Gemycircularvirus chicas 1 & $\begin{array}{l}\text { Chicken associated gemycircular- } \\
\text { virus } 1\end{array}$ & $\begin{array}{l}\text { Faeces associated gemycircularvirus } \\
20\end{array}$ & KT862243 \\
\hline & Gemycircularvirus chicas 2 & $\begin{array}{l}\text { Chicken associated gemycircular- } \\
\text { virus } 2\end{array}$ & $\begin{array}{l}\text { Faeces associated gemycircularvirus } \\
\quad 17\end{array}$ & KT862242 \\
\hline & Gemycircularvirus chickadl & $\begin{array}{l}\text { Chickadee associated gemycircular- } \\
\text { virus } 1\end{array}$ & $\begin{array}{l}\text { Poecile atricapillus GI tract-associ- } \\
\text { ated gemycircularvirus }\end{array}$ & KT309029 \\
\hline & Gemycircularvirus citas 1 & - & Citrus Tunisia genomovirus 2 & MN708485 \\
\hline & Gemycircularvirus cybusil & - & Spider associated circular virus 1 & MH545503 \\
\hline & Gemycircularvirus denbre1 & - & $\begin{array}{l}\text { Bark beetle-associated genomovi- } \\
\text { rus } 1\end{array}$ & MG571096 \\
\hline & Gemycircularvirus denbre 2 & - & $\begin{array}{l}\text { Bark beetle-associated genomovi- } \\
\text { rus } 2\end{array}$ & MG571098 \\
\hline & Gemycircularvirus denbre3 & - & $\begin{array}{l}\text { Bark beetle-associated genomovi- } \\
\text { rus } 3\end{array}$ & MG571099 \\
\hline & Gemycircularvirus denbre 4 & - & $\begin{array}{l}\text { Bark beetle-associated genomovi- } \\
\text { rus } 4\end{array}$ & MG571100 \\
\hline & Gemycircularvirus denpol & - & $\begin{array}{l}\text { Bark beetle-associated genomovi- } \\
\text { rus } 5\end{array}$ & MG571101 \\
\hline & Gemycircularvirus derval & - & Tick-associated genomovirus 1 & MF173065 \\
\hline & Gemycircularvirus dichism1 & - & Plant associated genomovirus 19 & MH939446 \\
\hline & Gemycircularvirus dragal & $\begin{array}{l}\text { Dragonfly associated gemycircular- } \\
\text { virus } 1\end{array}$ & $\begin{array}{l}\text { Dragonfly-associated circular virus } \\
2\end{array}$ & JX185429 \\
\hline & Gemycircularvirus echiam 1 & - & Thrips-associated genomovirus 1 & KY308268 \\
\hline \multirow[t]{3}{*}{ - } & Gemycircularvirus equas 1 & $\begin{array}{l}\text { Equine associated gemycircularvi- } \\
\text { rus } 1\end{array}$ & $\begin{array}{l}\text { Faeces associated gemycircularvirus } \\
\quad 18\end{array}$ & KT862248 \\
\hline & Gemycircularvirus eratil & - & $\begin{array}{l}\text { Giant house spider associated circu- } \\
\text { lar virus } 1\end{array}$ & MH545509 \\
\hline & Gemycircularvirus euhet 1 & - & $\begin{array}{l}\text { Euphorbia heterophylla associated } \\
\text { gemycircularvirus }\end{array}$ & MH047858 \\
\hline
\end{tabular}


Table 1 (continued)

\begin{tabular}{|c|c|c|c|c|}
\hline Genus & New species name & Former species name & Exemplar virus & Accession no. \\
\hline & Gemycircularvirus furse1 & $\begin{array}{l}\text { Fur seal associated gemycircular- } \\
\text { virus } 1\end{array}$ & $\begin{array}{l}\text { Faecal-associated gemycircularvi- } \\
\text { rus } 4\end{array}$ & KF371638 \\
\hline & Gemycircularvirus geras 1 & $\begin{array}{l}\text { Gerygone associated gemycircular- } \\
\text { virus } 1\end{array}$ & $\begin{array}{l}\text { Faecal-associated gemycircularvi- } \\
\text { rus } 6\end{array}$ & KF371636 \\
\hline & Gemycircularvirus geras 2 & $\begin{array}{l}\text { Gerygone associated gemycircular- } \\
\text { virus } 2\end{array}$ & $\begin{array}{l}\text { Faecal-associated gemycircularvi- } \\
\text { rus } 5\end{array}$ & KF371637 \\
\hline & Gemycircularvirus geras 3 & $\begin{array}{l}\text { Gerygone associated gemycircular- } \\
\text { virus } 3\end{array}$ & $\begin{array}{l}\text { Faecal-associated gemycircularvi- } \\
\text { rus } 3\end{array}$ & KF371639 \\
\hline & Gemycircularvirus giapal & - & $\begin{array}{l}\text { Giant panda associated gemycircu- } \\
\text { larvirus }\end{array}$ & MF327560 \\
\hline & Gemycircularvirus giapa2 & - & $\begin{array}{l}\text { Giant panda associated gemycircu- } \\
\text { larvirus }\end{array}$ & MF327565 \\
\hline & Gemycircularvirus giapa3 & - & $\begin{array}{l}\text { Giant panda associated gemycircu- } \\
\text { larvirus }\end{array}$ & MF327568 \\
\hline & Gemycircularvirus giapa4 & - & $\begin{array}{l}\text { Giant panda associated gemycircu- } \\
\text { larvirus }\end{array}$ & MF327561 \\
\hline & Gemycircularvirus giapa5 & - & $\begin{array}{l}\text { Giant panda associated gemycircu- } \\
\text { larvirus }\end{array}$ & MF327567 \\
\hline & Gemycircularvirus giapa6 & - & $\begin{array}{l}\text { Giant panda associated gemycircu- } \\
\text { larvirus }\end{array}$ & MF327569 \\
\hline & Gemycircularvirus giapa7 & - & $\begin{array}{l}\text { Giant panda associated gemycircu- } \\
\text { larvirus }\end{array}$ & MF327558 \\
\hline & Gemycircularvirus giapa8 & - & $\begin{array}{l}\text { Giant panda associated gemycircu- } \\
\text { larvirus }\end{array}$ & MF327570 \\
\hline & Gemycircularvirus gophal & - & Tortoise genomovirus 17 & MK570217 \\
\hline & Gemycircularvirus gopha2 & - & Tortoise genomovirus 10 & MK570210 \\
\hline & Gemycircularvirus gopha3 & - & Genomoviridae sp. & MK032737 \\
\hline & Gemycircularvirus hadtis1 & - & Genomoviridae sp. & MK032733 \\
\hline & Gemycircularvirus haeme1 & - & Finch associated genomovirus 5 & MK249235 \\
\hline & Gemycircularvirus haeme2 & - & Finch associated genomovirus 6 & MK249242 \\
\hline & Gemycircularvirus hydrol & - & Capybara genomovirus 1 & MK483072 \\
\hline & Gemycircularvirus hypas1 & $\begin{array}{l}\text { Hypericum associated gemycircu- } \\
\text { larvirus } 1\end{array}$ & $\begin{array}{l}\text { Hypericum japonicum associated } \\
\text { circular DNA virus }\end{array}$ & KF413620 \\
\hline & Gemycircularvirus ixode1 & - & Tick-associated genomovirus 3 & MF173067 \\
\hline & Gemycircularvirus lamas 1 & $\begin{array}{l}\text { Lama associated gemycircularvirus } \\
\quad 1\end{array}$ & $\begin{array}{l}\text { Faeces associated gemycircularvirus } \\
\quad 21\end{array}$ & KT862245 \\
\hline & Gemycircularvirus lebecl & - & Plant associated genomovirus 20 & MH939431 \\
\hline & Gemycircularvirus legle1 & - & Plant associated genomovirus 15 & MH939377 \\
\hline & Gemycircularvirus lepa 2 & - & $\begin{array}{l}\text { Lynx canadensis faeces associated } \\
\text { genomovirus CL1 } 46\end{array}$ & MG641191 \\
\hline & Gemycircularvirus lepam1 & - & $\begin{array}{l}\text { Alces alces faeces associated } \\
\text { genomovirus MP111 }\end{array}$ & MG641205 \\
\hline & Gemycircularvirus lepam 2 & - & $\begin{array}{l}\text { Alces alces faeces associated } \\
\text { genomovirus MP43 }\end{array}$ & MG641202 \\
\hline & Gemycircularvirus lepam3 & - & $\begin{array}{l}\text { Lepus americanus faeces associated } \\
\text { genomovirus SHP9 }\end{array}$ & MG641211 \\
\hline & Gemycircularvirus lyncal & - & $\begin{array}{l}\text { Lynx canadensis faeces associated } \\
\text { genomovirus CL1 } 48\end{array}$ & MG641192 \\
\hline & Gemycircularvirus lynca2 & - & $\begin{array}{l}\text { Lynx canadensis faeces associated } \\
\text { genomovirus CL1 } 71\end{array}$ & MG641194 \\
\hline & Gemycircularvirus lynca 3 & - & $\begin{array}{l}\text { Lynx canadensis faeces associated } \\
\text { genomovirus CL5 } 48\end{array}$ & MG641201 \\
\hline & Gemycircularvirus lynca4 & - & $\begin{array}{l}\text { Lynx canadensis faeces associated } \\
\text { genomovirus CL1 } 148\end{array}$ & MG641197 \\
\hline
\end{tabular}


Table 1 (continued)

\begin{tabular}{|c|c|c|c|c|}
\hline Genus & New species name & Former species name & Exemplar virus & Accession no. \\
\hline & Gemycircularvirus malas 1 & $\begin{array}{l}\text { Mallard associated gemycircular- } \\
\text { virus } 1\end{array}$ & $\begin{array}{l}\text { Faecal-associated gemycircularvi- } \\
\text { rus } 7\end{array}$ & KF371635 \\
\hline & Gemycircularvirus miniol & $\begin{array}{l}\text { Miniopterus associated gemycircu- } \\
\text { larvirus } 1\end{array}$ & Bat circovirus & KJ641719 \\
\hline & Gemycircularvirus minitil & - & Genomoviridae sp. & MK032736 \\
\hline & Gemycircularvirus miniti2 & - & Genomoviridae sp. & MK032701 \\
\hline & Gemycircularvirus miniti3 & - & Genomoviridae sp. & MK032712 \\
\hline & Gemycircularvirus miniti4 & - & Genomoviridae sp. & MK032709 \\
\hline & Gemycircularvirus minti6 & - & Genomoviridae sp. & MH617534 \\
\hline & Gemycircularvirus mochal & - & $\begin{array}{l}\text { Momordica charantia associated } \\
\text { gemycircularvirus }\end{array}$ & MH047857 \\
\hline & Gemycircularvirus monas 1 & $\begin{array}{l}\text { Mongoose associated gemycircular- } \\
\text { virus } 1\end{array}$ & $\begin{array}{l}\text { Mongoose feces-associated gemycir- } \\
\text { cularvirus d }\end{array}$ & KP263547 \\
\hline & Gemycircularvirus mosqil & $\begin{array}{l}\text { Mosquito associated gemycircular- } \\
\text { virus } 1\end{array}$ & Mosquito VEM virus SDBVL G & HQ335086 \\
\hline & Gemycircularvirus moutil & - & Genomoviridae sp. & MK032728 \\
\hline & Gemycircularvirus moutilo & - & Genomoviridae sp. & MK032705 \\
\hline & Gemycircularvirus moutil1 & - & Genomoviridae sp. & MK032718 \\
\hline & Gemycircularvirus moutil2 & - & Genomoviridae sp. & MK032702 \\
\hline & Gemycircularvirus mouti2 & - & Genomoviridae sp. & MK032755 \\
\hline & Gemycircularvirus mouti3 & - & Genomoviridae sp. & MK032715 \\
\hline & Gemycircularvirus mouti4 & - & Genomoviridae sp. & MK032719 \\
\hline & Gemycircularvirus mouti5 & - & Genomoviridae sp. & MK032753 \\
\hline & Gemycircularvirus mouti6 & - & Genomoviridae sp. & MK032735 \\
\hline & Gemycircularvirus mouti7 & - & Genomoviridae sp. & MK032738 \\
\hline & Gemycircularvirus mouti8 & - & Genomoviridae sp. & MK032752 \\
\hline & Gemycircularvirus mouti9 & - & Genomoviridae sp. & MK032714 \\
\hline & Gemycircularvirus odonal & $\begin{array}{l}\text { Odonata associated gemycircular- } \\
\text { virus } 1\end{array}$ & $\begin{array}{l}\text { Odonata associated gemycircular- } \\
\text { virus-1 }\end{array}$ & KM598385 \\
\hline & Gemycircularvirus odona 2 & $\begin{array}{l}\text { Odonata associated gemycircular- } \\
\text { virus } 2\end{array}$ & $\begin{array}{l}\text { Odonata associated gemycircular- } \\
\text { virus-2 }\end{array}$ & KM598387 \\
\hline & Gemycircularvirus oltre1 & - & $\begin{array}{l}\text { Olive associated gemycircularvirus } \\
1\end{array}$ & MH444690 \\
\hline & Gemycircularvirus opunt1 & - & Plant associated genomovirus 25 & MK947372 \\
\hline & Gemycircularvirus oxcor1 & - & Oxalis corniculata genomoviridae & MN823668 \\
\hline & Gemycircularvirus plecal & - & Gemycircularvirus sp. & KY302866 \\
\hline & Gemycircularvirus poass 1 & $\begin{array}{l}\text { Poaceae associated gemycircular- } \\
\text { virus } 1\end{array}$ & $\begin{array}{l}\text { Poaceae-associated gemycircular- } \\
\text { virus } 1\end{array}$ & KT253577 \\
\hline & Gemycircularvirus porcil & $\begin{array}{l}\text { Porcine associated gemycircular- } \\
\text { virus } 1\end{array}$ & $\begin{array}{l}\text { Faeces associated gemycircularvirus } \\
\quad 19\end{array}$ & KT862250 \\
\hline & Gemycircularvirus porci2 & $\begin{array}{l}\text { Porcine associated gemycircular- } \\
\text { virus } 2\end{array}$ & $\begin{array}{l}\text { Faecal-associated gemycircularvi- } \\
\text { rus } 2\end{array}$ & KF371640 \\
\hline & Gemycircularvirus pterol & $\begin{array}{l}\text { Pteropus associated gemycircular- } \\
\text { virus } 1\end{array}$ & $\begin{array}{l}\text { Pacific flying fox faeces associated } \\
\text { gemycircularvirus-10 }\end{array}$ & KT732804 \\
\hline & Gemycircularvirus ptero10 & $\begin{array}{l}\text { Pteropus associated gemycircular- } \\
\text { virus } 10\end{array}$ & $\begin{array}{l}\text { Pacific flying fox faeces associated } \\
\text { gemycircularvirus- } 3\end{array}$ & KT732794 \\
\hline & Gemycircularvirus ptero 2 & $\begin{array}{l}\text { Pteropus associated gemycircular- } \\
\text { virus } 2\end{array}$ & $\begin{array}{l}\text { Pacific flying fox faeces associated } \\
\text { gemycircularvirus- } 2\end{array}$ & KT732792 \\
\hline & Gemycircularvirus ptero3 & $\begin{array}{l}\text { Pteropus associated gemycircular- } \\
\text { virus } 3\end{array}$ & $\begin{array}{l}\text { Pacific flying fox faeces associated } \\
\text { gemycircularvirus-5 }\end{array}$ & KT732797 \\
\hline & Gemycircularvirus ptero 4 & $\begin{array}{l}\text { Pteropus associated gemycircular- } \\
\text { virus } 4\end{array}$ & $\begin{array}{l}\text { Pacific flying fox faeces associated } \\
\text { gemycircularvirus-13 }\end{array}$ & KT732814 \\
\hline
\end{tabular}


Table 1 (continued)

\begin{tabular}{|c|c|c|c|c|}
\hline Genus & New species name & Former species name & Exemplar virus & Accession no. \\
\hline & Gemycircularvirus ptero5 & $\begin{array}{l}\text { Pteropus associated gemycircular- } \\
\text { virus } 5\end{array}$ & $\begin{array}{l}\text { Pacific flying fox faeces associated } \\
\text { gemycircularvirus- } 8\end{array}$ & KT732801 \\
\hline & Gemycircularvirus ptero6 & $\begin{array}{l}\text { Pteropus associated gemycircular- } \\
\text { virus } 6\end{array}$ & $\begin{array}{l}\text { Pacific flying fox faeces associated } \\
\text { gemycircularvirus- } 9\end{array}$ & KT732803 \\
\hline & Gemycircularvirus ptero 7 & $\begin{array}{l}\text { Pteropus associated gemycircular- } \\
\text { virus } 7\end{array}$ & $\begin{array}{l}\text { Pacific flying fox faeces associated } \\
\text { gemycircularvirus-11 }\end{array}$ & KT732807 \\
\hline & Gemycircularvirus ptero8 & $\begin{array}{l}\text { Pteropus associated gemycircular- } \\
\text { virus } 8\end{array}$ & $\begin{array}{l}\text { Pacific flying fox faeces associated } \\
\text { gemycircularvirus-14 }\end{array}$ & KT732806 \\
\hline & Gemycircularvirus ptero 9 & $\begin{array}{l}\text { Pteropus associated gemycircular- } \\
\text { virus } 9\end{array}$ & $\begin{array}{l}\text { Pacific flying fox faeces associated } \\
\text { gemycircularvirus- } 4\end{array}$ & KT732795 \\
\hline & Gemycircularvirus raskil & - & Genomoviridae sp. & MK032754 \\
\hline & Gemycircularvirus ratas 1 & Rat associated gemycircularvirus 1 & Gemycircularvirus gemy-ch-rat1 & KR912221 \\
\hline & Gemycircularvirus rebac1 & - & $\begin{array}{l}\text { Northern red-backed vole stool- } \\
\text { associated gemycircularvirus } 110\end{array}$ & MK738141 \\
\hline & Gemycircularvirus recrol & - & Gemycircularvirus sp. & KY312557 \\
\hline & Gemycircularvirus sarpe1 & - & Plant associated genomovirus 17 & MH939397 \\
\hline & Gemycircularvirus sclerol & Sclerotinia gemycircularvirus 1 & $\begin{array}{l}\text { Sclerotinia sclerotiorum hypoviru- } \\
\text { lence associated DNA virus } 1\end{array}$ & GQ365709 \\
\hline & Gemycircularvirus sewopol & Sewage derived gemycircularvirus 1 & $\begin{array}{l}\text { Sewage-associated gemycircular- } \\
\text { virus- } 8\end{array}$ & KJ547638 \\
\hline & Gemycircularvirus sewopo2 & Sewage derived gemycircularvirus 2 & $\begin{array}{l}\text { Sewage-associated gemycircularvi- } \\
\text { rus-11 }\end{array}$ & KJ547641 \\
\hline & Gemycircularvirus sewopo3 & Sewage derived gemycircularvirus 3 & $\begin{array}{l}\text { Sewage-associated gemycircular- } \\
\text { virus- } 6\end{array}$ & KJ547636 \\
\hline & Gemycircularvirus sewopo4 & Sewage derived gemycircularvirus 4 & $\begin{array}{l}\text { Sewage-associated gemycircularvi- } \\
\text { rus- } 7 \mathrm{~b}\end{array}$ & KJ547640 \\
\hline & Gemycircularvirus sewopo5 & Sewage derived gemycircularvirus 5 & $\begin{array}{l}\text { Sewage-associated gemycircular- } \\
\text { virus-9 }\end{array}$ & KJ547639 \\
\hline & Gemycircularvirus sheas 1 & $\begin{array}{l}\text { Sheep associated gemycircularvirus } \\
1\end{array}$ & $\begin{array}{l}\text { Faeces associated gemycircularvirus } \\
16\end{array}$ & KT862249 \\
\hline & Gemycircularvirus siedol & - & $\begin{array}{l}\text { Sierra dome spider associated circu- } \\
\text { lar virus } 1\end{array}$ & MH545510 \\
\hline & Gemycircularvirus solas 1 & - & Plant associated genomovirus 11 & MH939384 \\
\hline & Gemycircularvirus soybe1 & $\begin{array}{l}\text { Soybean associated gemycircular- } \\
\text { virus } 1\end{array}$ & $\begin{array}{l}\text { Soybean leaf-associated gemycircu- } \\
\text { larvirus } 1\end{array}$ & KT598248 \\
\hline & Gemycircularvirus termil & - & Termite associated circular virus 2 & MG917675 \\
\hline & Gemycircularvirus trilo1 & - & Plant associated genomovirus 22 & MH939442 \\
\hline & Gemycircularvirus turtil & - & Genomoviridae sp. & MK012473 \\
\hline & Gemycircularvirus willde1 & - & Plant associated genomovirus 13 & МH939427 \\
\hline \multirow[t]{12}{*}{ Gemyduguivirus } & Gemyduguivirus artecal & - & Artemisia carvifolia genomoviridae & MN823676 \\
\hline & Gemyduguivirus austol & - & Blackfly genomovirus 8 & MK433240 \\
\hline & Gemyduguivirus bemtal & - & $\begin{array}{l}\text { Bemisia-associated genomovirus } \\
\text { AdDF }\end{array}$ & KY230613 \\
\hline & Gemyduguivirus dragal & $\begin{array}{l}\text { Dragonfly associated gemyduguivi- } \\
\quad \text { rus } 1\end{array}$ & $\begin{array}{l}\text { Dragonfly-associated circular virus } \\
3\end{array}$ & JX185428 \\
\hline & Gemyduguivirus hydrol & - & Capybara genomovirus 3 & MK483075 \\
\hline & Gemyduguivirus hydro2 & - & Capybara genomovirus 8 & MK483080 \\
\hline & Gemyduguivirus hydro3 & - & Capybara genomovirus 5 & MK483077 \\
\hline & Gemyduguivirus macral & - & Plant associated genomovirus 4 & МH939370 \\
\hline & Gemyduguivirus merre1 & - & Plant associated genomovirus 5 & MH939417 \\
\hline & Gemyduguivirus mintil & - & Genomoviridae sp. & MK032731 \\
\hline & Gemyduguivirus minti2 & - & Genomoviridae sp. & MK032726 \\
\hline & Gemyduguivirus recrol & - & Gemycircularvirus sp. & KY312558 \\
\hline
\end{tabular}


Table 1 (continued)

\begin{tabular}{|c|c|c|c|c|}
\hline Genus & New species name & Former species name & Exemplar virus & Accession no. \\
\hline \multirow[t]{8}{*}{ Gemygorvirus } & Gemygorvirus canial & Canine associated gemygorvirus 1 & $\begin{array}{l}\text { Faeces associated gemycircularvirus } \\
\quad 15\end{array}$ & KT862254 \\
\hline & Gemygorvirus hydrol & - & Capybara genomovirus 6 & MK483078 \\
\hline & Gemygorvirus malas1 & Mallard associated gemygorvirus 1 & $\begin{array}{l}\text { Faeces associated gemycircularvirus } \\
14\end{array}$ & KT862238 \\
\hline & Gemygorvirus opunt1 & - & Plant associated genomovirus 26 & MK947373 \\
\hline & Gemygorvirus poaspe1 & - & Plant associated genomovirus 1 & MH939361 \\
\hline & Gemygorvirus pterol & Pteropus associated gemygorvirus 1 & $\begin{array}{l}\text { Pacific flying fox faeces associated } \\
\text { gemycircularvirus-1 }\end{array}$ & KT732790 \\
\hline & Gemygorvirus sewopol & Sewage derived gemygorvirus 1 & $\begin{array}{l}\text { Sewage-associated gemycircular- } \\
\text { virus-5 }\end{array}$ & KJ547635 \\
\hline & Gemygorvirus staral & Starling associated gemygorvirus 1 & $\begin{array}{l}\text { Faecal-associated gemycircularvirus } \\
\quad 10\end{array}$ & KF371632 \\
\hline \multirow[t]{30}{*}{ Gemykibivirus } & Gemykibivirus abatil & - & Genomoviridae sp. & MK032696 \\
\hline & Gemykibivirus abati2 & - & Genomoviridae sp. & MK032759 \\
\hline & Gemykibivirus animal & - & $\begin{array}{l}\text { Porcine feces-associated gemycircu- } \\
\text { larvirus }\end{array}$ & KY214433 \\
\hline & Gemykibivirus badas1 & Badger associated gemykibivirus 1 & $\begin{array}{l}\text { Badger feces-associated gemycircu- } \\
\text { larvirus }\end{array}$ & KP263543 \\
\hline & Gemykibivirus bemtal & - & $\begin{array}{l}\text { Bemisia-associated genomovirus } \\
\text { NfO }\end{array}$ & KY230625 \\
\hline & Gemykibivirus blabil & $\begin{array}{l}\text { Blackbird associated gemykibivirus } \\
1\end{array}$ & $\begin{array}{l}\text { Faecal-associated gemycircularvi- } \\
\text { rus } 9\end{array}$ & KF371633 \\
\hline & Gemykibivirus blarol & $\begin{array}{l}\text { Black robin associated gemykibivi- } \\
\text { rus } 1\end{array}$ & $\begin{array}{l}\text { Faecal-associated gemycircularvi- } \\
\text { rus } 8\end{array}$ & KF371634 \\
\hline & Gemykibivirus bovas 1 & Bovine associated gemykibivirus 1 & HCBI8.215 virus & LK931483 \\
\hline & Gemykibivirus canfam 1 & - & $\begin{array}{l}\text { Canine feces-associated gemycircu- } \\
\text { larvirus }\end{array}$ & KY214441 \\
\hline & Gemykibivirus cowchil & - & $\begin{array}{l}\text { Cattle blood-associated gemycircu- } \\
\text { larvirus }\end{array}$ & MF669480 \\
\hline & Gemykibivirus cybusil & - & $\begin{array}{l}\text { Cybaeus spider associated circular } \\
\text { virus } 2\end{array}$ & MH545507 \\
\hline & Gemykibivirus cynas 1 & - & Plant associated genomovirus 3 & MH939438 \\
\hline & Gemykibivirus dragal & $\begin{array}{l}\text { Dragonfly associated gemykibivirus } \\
\quad 1\end{array}$ & $\begin{array}{l}\text { Dragonfly-associated circular virus } \\
1\end{array}$ & JX185430 \\
\hline & Gemykibivirus echil & - & Thrips-associated genomovirus 3 & KY308269 \\
\hline & Gemykibivirus galgal & - & Chicken genomovirus mg7_73 & MN379612 \\
\hline & Gemykibivirus galga2 & - & Chicken genomovirus mg8_401 & MN379615 \\
\hline & Gemykibivirus galga3 & - & Chicken genomovirus mg4_1218 & MN379608 \\
\hline & Gemykibivirus giapal & - & $\begin{array}{l}\text { Giant panda associated gemycircu- } \\
\text { larvirus }\end{array}$ & MF327571 \\
\hline & Gemykibivirus hadtis1 & - & Genomoviridae sp. & MK032708 \\
\hline & Gemykibivirus haeme1 & - & Finch associated genomovirus 3 & MK249305 \\
\hline & Gemykibivirus haeme2 & - & Gopherus associated genomovirus 1 & MF373638 \\
\hline & Gemykibivirus haeme3 & - & Finch associated genomovirus 2 & MK249239 \\
\hline & Gemykibivirus haeme4 & - & Finch associated genomovirus 4 & MK249240 \\
\hline & Gemykibivirus haeme5 & - & Finch associated genomovirus 1 & MK249294 \\
\hline & Gemykibivirus hiplal & - & Bat gemycircularvirus & MK050014 \\
\hline & Gemykibivirus humasl & Human associated gemykibivirus 1 & MSSI2.225 virus & LK931485 \\
\hline & Gemykibivirus humas2 & Human associated gemykibivirus 2 & Gemycircularvirus SL1 & KP133075 \\
\hline & Gemykibivirus humas3 & Human associated gemykibivirus 3 & Gemycircularvirus C1c & KP987887 \\
\hline & Gemykibivirus humas4 & Human associated gemykibivirus 4 & Human gemycircularvirus GeTz1 & KT363839 \\
\hline & Gemykibivirus humas5 & Human associated gemykibivirus 5 & Gemycircularvirus HV-GcV2 & KU343137 \\
\hline
\end{tabular}


Table 1 (continued)

\begin{tabular}{|c|c|c|c|c|}
\hline Genus & New species name & Former species name & Exemplar virus & Accession no. \\
\hline & Gemykibivirus hydrol & - & Capybara genomovirus 4 & MK483076 \\
\hline & Gemykibivirus hydro2 & - & Capybara genomovirus 2 & MK483073 \\
\hline & Gemykibivirus hydro3 & - & Capybara genomovirus 12 & MK483084 \\
\hline & Gemykibivirus mintil & - & Genomoviridae sp. & MK032742 \\
\hline & Gemykibivirus monas 1 & $\begin{array}{l}\text { Mongoose associated gemykibivirus } \\
\quad 1\end{array}$ & $\begin{array}{l}\text { Mongoose feces-associated gemycir- } \\
\text { cularvirus b }\end{array}$ & KP263545 \\
\hline & Gemykibivirus moutil & - & Genomoviridae sp. & MK032703 \\
\hline & Gemykibivirus mouti2 & - & Genomoviridae sp. & MK032748 \\
\hline & Gemykibivirus pitis1 & - & Genomoviridae sp. & MK032749 \\
\hline & Gemykibivirus pitis2 & - & Genomoviridae sp. & MK032721 \\
\hline & Gemykibivirus plantal & - & Plant associated genomovirus 2 & MH939363 \\
\hline & Gemykibivirus planta2 & - & Plant associated genomovirus 29 & MK947376 \\
\hline & Gemykibivirus pterol & Pteropus associated gemykibivirus 1 & $\begin{array}{l}\text { Pacific flying fox faeces associated } \\
\text { gemycircularvirus- } 12\end{array}$ & KT732813 \\
\hline & Gemykibivirus raskil & - & Genomoviridae sp. & MK032704 \\
\hline & Gemykibivirus rhinal & $\begin{array}{l}\text { Rhinolophus associated gemykibi- } \\
\text { virus } 1\end{array}$ & Bat circovirus & KJ641737 \\
\hline & Gemykibivirus rhina 2 & $\begin{array}{l}\text { Rhinolophus associated gemykibi- } \\
\text { virus } 2\end{array}$ & Bat circovirus & KJ641726 \\
\hline & Gemykibivirus sewopol & Sewage derived gemykibivirus 1 & $\begin{array}{l}\text { Sewage-associated gemycircular- } \\
\text { virus-3 }\end{array}$ & KJ547643 \\
\hline & Gemykibivirus sewopo 2 & Sewage derived gemykibivirus 2 & $\begin{array}{l}\text { Sewage-associated gemycircular- } \\
\text { virus-2 }\end{array}$ & KJ547642 \\
\hline & Gemykibivirus turtil & - & Genomoviridae sp. & MK012443 \\
\hline & Gemykibivirus wastel & - & Genomoviridae sp. & MT309857 \\
\hline & Gemykibivirus womot1 & - & Genomoviridae sp. & MK032756 \\
\hline \multirow[t]{16}{*}{ Gemykolovirus } & Gemykolovirus abatil & - & Genomoviridae sp. & MK032717 \\
\hline & Gemykolovirus citas 1 & - & Citrus Tunisia genomovirus $1 \mathrm{~b}$ & MN708482 \\
\hline & Gemykolovirus derval & - & Tick-associated genomovirus 2 & MF173066 \\
\hline & Gemykolovirus easlu1 & - & $\begin{array}{l}\text { Grasshopper associated circular } \\
\text { virus } 1\end{array}$ & MH545499 \\
\hline & Gemykolovirus echial & - & Thrips-associated genomovirus 4 & KY308270 \\
\hline & Gemykolovirus gophal & - & Tortoise genomovirus 9 & MK570209 \\
\hline & Gemykolovirus gopha2 & - & Tortoise genomovirus 13 & MK570213 \\
\hline & Gemykolovirus hadtis1 & - & Genomoviridae sp. & MK032747 \\
\hline & Gemykolovirus herisl & - & Plant associated genomovirus 7 & МH939374 \\
\hline & Gemykolovirus lepam1 & - & $\begin{array}{l}\text { Alces alces faeces associated } \\
\text { genomovirus MP68 }\end{array}$ & MG641203 \\
\hline & Gemykolovirus poaspe1 & - & Plant associated genomovirus 9 & MH939382 \\
\hline & Gemykolovirus prupel & - & Amygdalus persica genomoviridae & MN823669 \\
\hline & Gemykolovirus pterol & Pteropus associated gemykolovirus 1 & $\begin{array}{l}\text { Pacific flying fox faeces associated } \\
\text { gemycircularvirus-6 }\end{array}$ & KT732798 \\
\hline & Gemykolovirus ptero2 & Pteropus associated gemykolovirus 2 & $\begin{array}{l}\text { Pacific flying fox faeces associated } \\
\text { gemycircularvirus-7 }\end{array}$ & KT732800 \\
\hline & Gemykolovirus segpal & - & $\begin{array}{l}\text { Tubeweb spider associated circular } \\
\text { virus } 1\end{array}$ & MH545501 \\
\hline & Gemykolovirus trotil & - & Genomoviridae sp. & MK032739 \\
\hline \multirow[t]{4}{*}{ Gemykrogvirus } & Gemykrogvirus abatil & - & Genomoviridae sp. & MK032716 \\
\hline & Gemykrogvirus apime1 & - & Apis mellifera genomovirus 2 & MH973741 \\
\hline & Gemykrogvirus bovas 1 & Bovine associated gemykrogvirus 1 & HCBI9.212 virus & LK931484 \\
\hline & Gemykrogvirus caribl & Caribou associated gemykrogvirus 1 & $\begin{array}{l}\text { Caribou feces-associated gemycir- } \\
\text { cularvirus }\end{array}$ & KJ938717 \\
\hline
\end{tabular}


Table 1 (continued)

\begin{tabular}{|c|c|c|c|c|}
\hline Genus & New species name & Former species name & Exemplar virus & Accession no. \\
\hline & Gemykrogvirus galgal & - & Chicken genomovirus mg4_1247 & MN379609 \\
\hline & Gemykrogvirus galga 2 & - & $\begin{array}{l}\text { Chicken stool-associated gemycir- } \\
\text { cularvirus }\end{array}$ & KY056250 \\
\hline & Gemykrogvirus galga3 & - & Chicken genomovirus mg4_1173 & MN379605 \\
\hline & Gemykrogvirus galga4 & - & Chicken genomovirus mg7_78 & MN379614 \\
\hline & Gemykrogvirus galga5 & - & Chicken genomovirus mg4_1165 & MN379604 \\
\hline & Gemykrogvirus giapal & - & $\begin{array}{l}\text { Giant panda associated gemycircu- } \\
\text { larvirus }\end{array}$ & MF327559 \\
\hline & Gemykrogvirus hadtis1 & - & Genomoviridae sp. & MK032724 \\
\hline & Gemykrogvirus humas1 & - & Gemycircularvirus sp. & MT649486 \\
\hline & Gemykrogvirus sewopol & Sewage derived gemykrogvirus 1 & $\begin{array}{l}\text { Sewage-associated gemycircular- } \\
\text { virus- } 4\end{array}$ & KJ547634 \\
\hline \multirow[t]{7}{*}{ Gemykronzavirus } & Gemykronzavirus animal & - & Genomoviridae sp. & MK032727 \\
\hline & Gemykronzavirus haeme1 & - & Finch associated genomovirus 8 & MK249245 \\
\hline & Gemykronzavirus hydrol & - & Capybara genomovirus 10 & MK483082 \\
\hline & Gemykronzavirus poaspe1 & - & Plant associated genomovirus 24 & MH939435 \\
\hline & Gemykronzavirus rabas 1 & $\begin{array}{l}\text { Rabbit associated gemykroznavirus } \\
\quad 1\end{array}$ & $\begin{array}{l}\text { Faecal-associated gemycircularvirus } \\
\quad 11\end{array}$ & KF371631 \\
\hline & Gemykronzavirus solas1 & - & Plant associated genomovirus 23 & MH939385 \\
\hline & Gemykronzavirus zizanl & - & Zizania latifolia genomoviridae & MN823671 \\
\hline Gemytondvirus & Gemytondvirus ostril & Ostrich associated gemytondvirus 1 & $\begin{array}{l}\text { Faecal-associated gemycircularvirus } \\
\quad 12\end{array}$ & KF371630 \\
\hline Gemytripvirus & Gemytripvirus fugral & - & $\begin{array}{l}\text { Fusarium graminearum gemytrip- } \\
\text { virus } 1\end{array}$ & $\begin{array}{c}\text { MK430076, } \\
\text { MK430077, } \\
\text { MK430078 }\end{array}$ \\
\hline Gemyvongvirus & Gemyvongvirus humas1 & Human associated gemyvongvirus 1 & $\begin{array}{l}\text { Human plasma-associated gemycir- } \\
\text { cularvirus }\end{array}$ & KP974693 \\
\hline \multirow[t]{2}{*}{ Gemyvongvirus } & Gemyvongvirus minit1 & - & Genomoviridae sp. & MK032710 \\
\hline & Gemyvongvirus minit 2 & - & Genomoviridae sp. & MK032740 \\
\hline
\end{tabular}




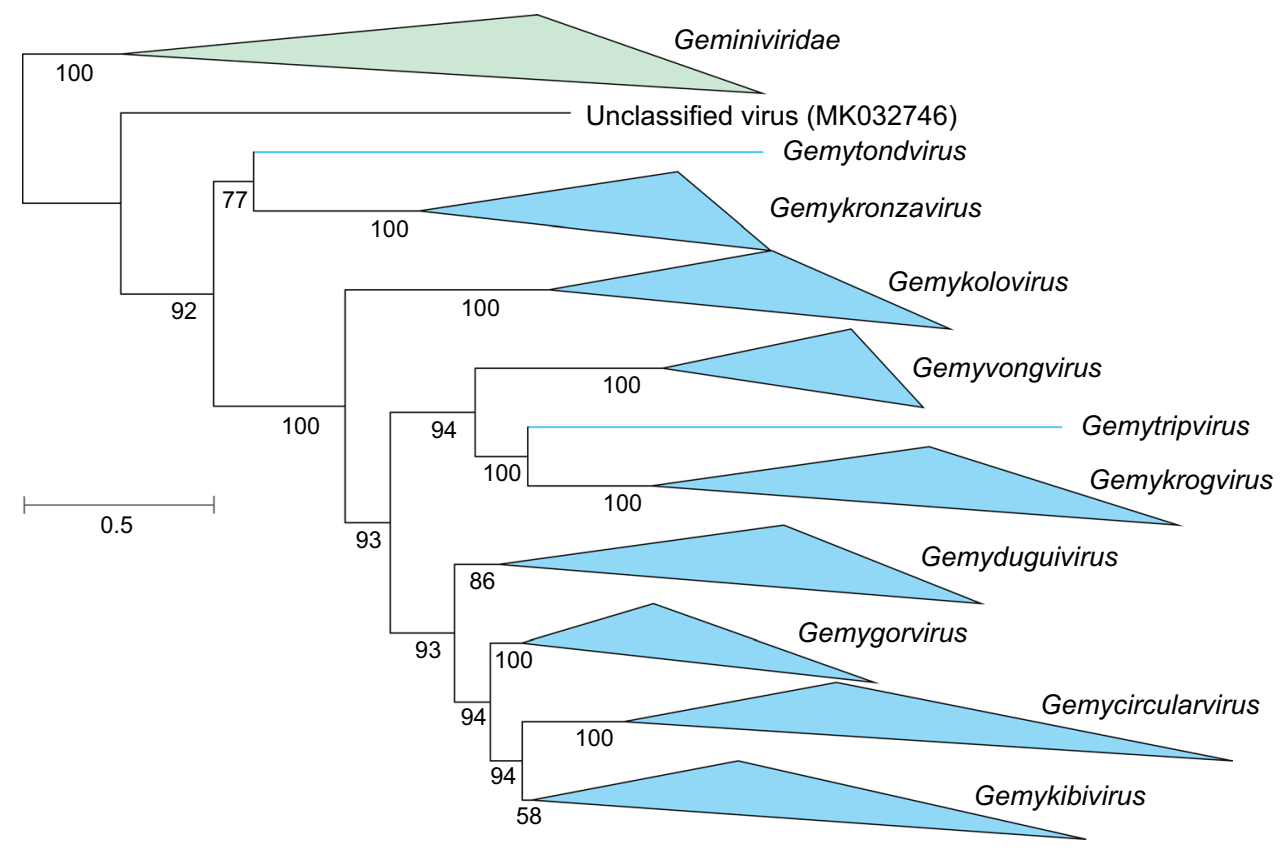

Fig. 1 Maximum-likelihood phylogenetic tree of Rep amino acid sequences of 545 genomoviruses together with a subset of geminiviruses and an unclassified virus (MK032746) that are distantly related. The tree is rooted with geminivirus sequences (green) and that of MK032746, which is most closely related to classified members of the family Genomoviridae. Clades corresponding to different genomovirus genera are colored blue. The Rep sequence alignment was con- structed with MAFFT [20] and trimmed with TrimAL [1] using the gappyout option. The final alignment contained 435 amino acid sites and was used to construct a maximum-likelihood phylogenetic tree using IQ-Tree [47]. The best-fitting model was determined by ModelFinder [19] and was LG+F+R9. Numbers at the nodes represent bootstrap support values (\%). 


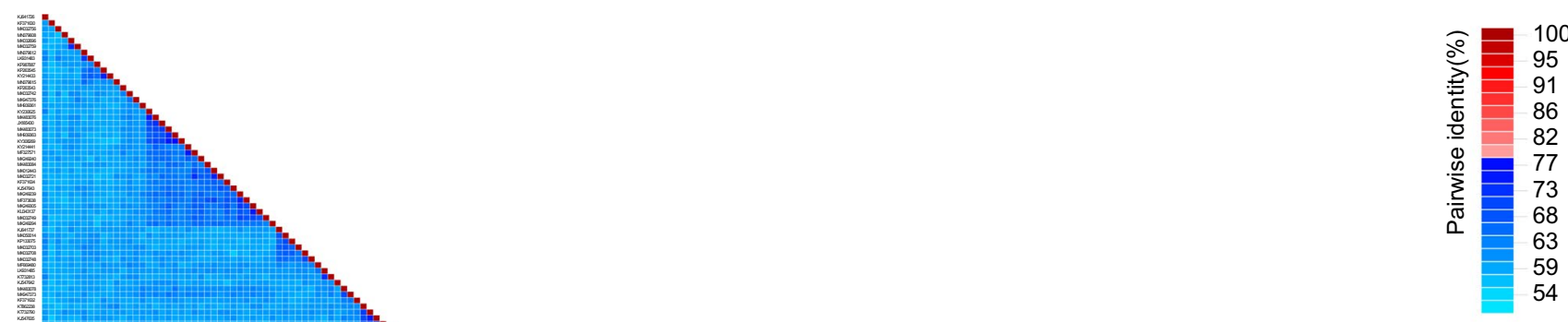

Fig. 2 Pairwise identity matrix of the genome sequence of a representative member of each species of genomovirus $(n=236)$ except Gemytripvirus fugral, whose representative, Fusarium graminearum gemytripvirus 1 , has a multicomponent genome. The analysis was performed using SDT v1.2 [49]. 


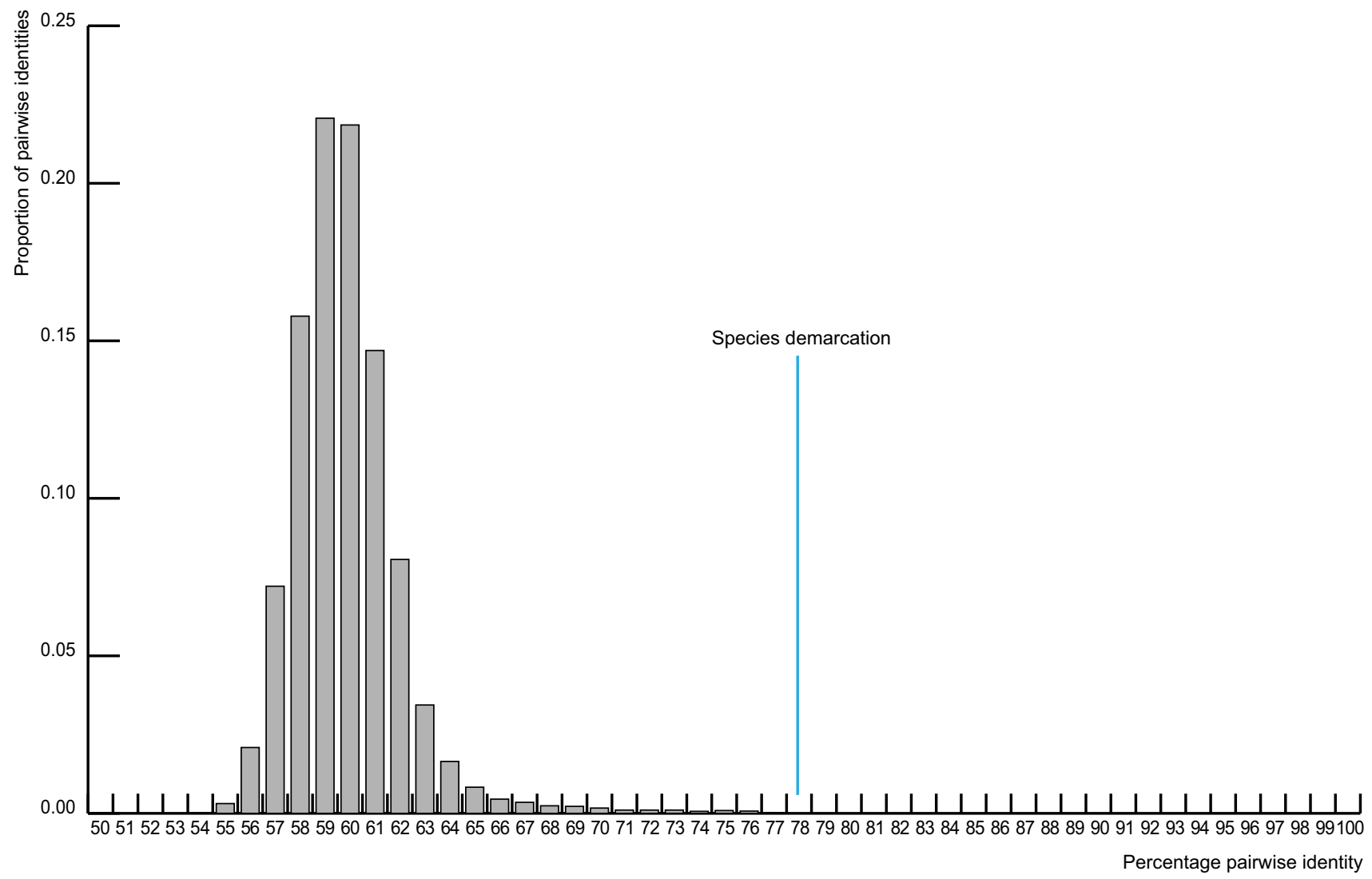

Fig. 3 Pairwise distribution plot of the 236 representative sequences of genomoviruses (except Fusarium graminearum gemytripvirus 1 [species Gemytripvirus fugral], which has a multicomponent genome), showing that no sequences from different species share $>78 \%$ identity. 
Fig. 4 Summary of the number of genomovirids assigned to each genus and source from which the genomes was obtained

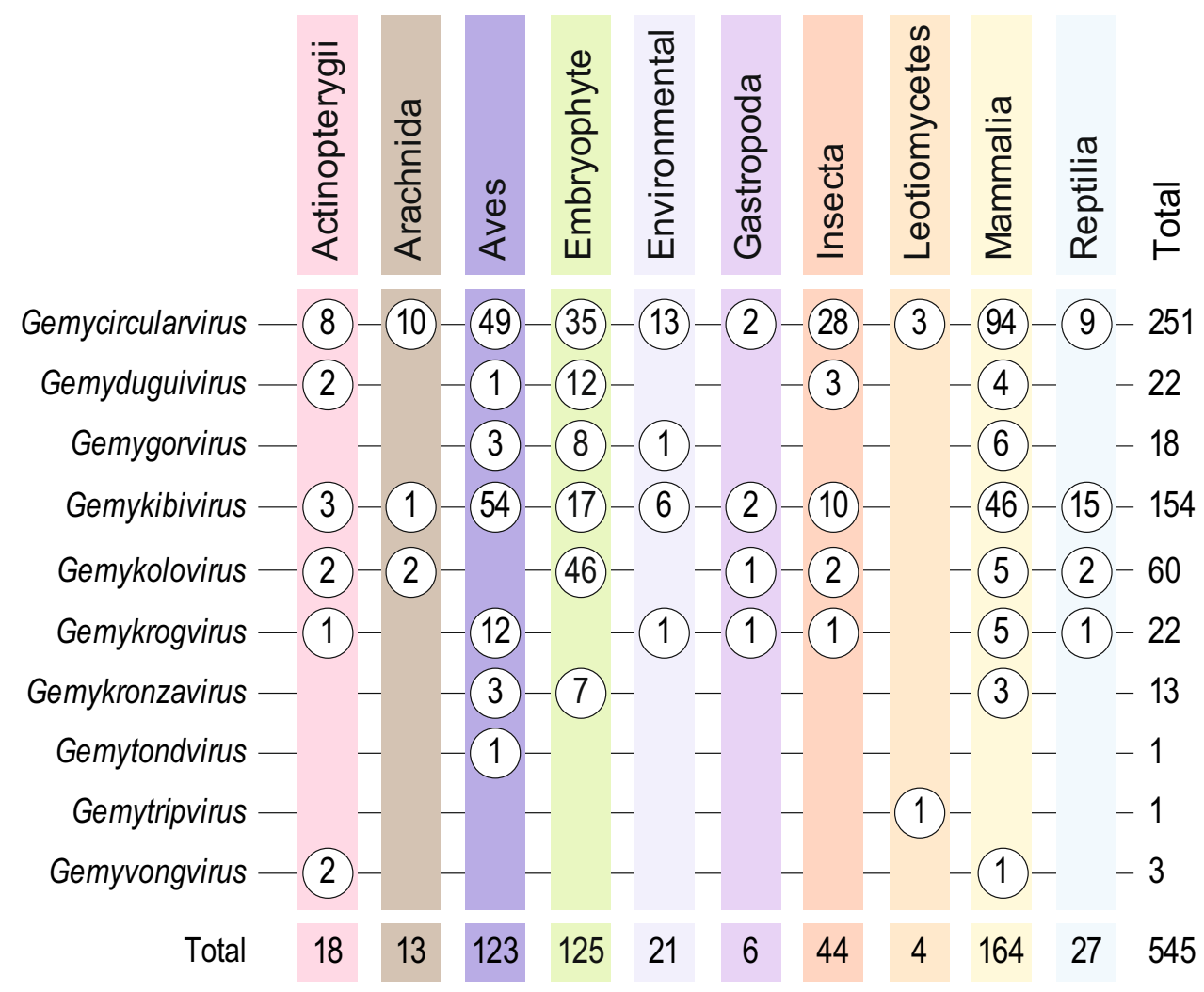

Supplementary Information The online version contains supplementary material available at https://doi.org/10.1007/s00705-021-05183-y.

Acknowledgements M.K. was supported by l'Agence Nationale de la Recherche (grant ANR-20-CE20-0009-02) and Ville de Paris (Emergence(s) project MEMREMA).

\section{Declarations}

Conflict of interest The authors declare no conflicts of interest.

\section{References}

1. Capella-Gutierrez S, Silla-Martinez JM, Gabaldon T (2009) tri$\mathrm{mAl}$ : a tool for automated alignment trimming in large-scale phylogenetic analyses. Bioinformatics 25:1972-1973

2. Chabi-Jesus C, Najar A, Fontenele RS, Kumari SG, Ramos-Gonzalez PL, Freitas-Astua J, Kraberger S, Varsani A (2020) Viruses representing two new genomovirus species identified in citrus from Tunisia. Arch Virol 165:1225-1229

3. Chiumenti M, Greco C, Antelmi I, Sion V, Altamura G, Nigro F, Saldarelli P (2019) Molecular characterisation of a novel gemycircularvirus associated with olive trees in Italy. Virus Res 263:169-172

4. Cibulski S, Alves de Lima D, Fernandes Dos Santos H, Teixeira TF, Tochetto C, Mayer FQ, Roehe PM (2021) A plate of viruses: viral metagenomics of supermarket chicken, pork and beef from Brazil. Virology 552:1-9

5. Cibulski SP, de Sales Lima FE, Teixeira TF, Varela APM, Scheffer CM, Mayer FQ, Witt AA, Roehe PM (2021) Detection of multiple viruses in oropharyngeal samples from Brazilian free-tailed bats (Tadarida brasiliensis) using viral metagenomics. Arch Virol 166:207-212

6. Conceicao-Neto N, Zeller M, Heylen E, Lefrere H, Mesquita JR, Matthijnssens J (2015) Fecal virome analysis of three carnivores reveals a novel nodavirus and multiple gemycircularviruses. Virol J 12:79

7. Conceicao-Neto N, Godinho R, Alvares F, Yinda CK, Deboutte W, Zeller M, Laenen L, Heylen E, Roque S, Petrucci-Fonseca F, Santos N, Van Ranst M, Mesquita JR, Matthijnssens J (2017) Viral gut metagenomics of sympatric wild and domestic canids, and monitoring of viruses: insights from an endangered wolf population. Ecol Evol 7:4135-4146

8. Dayaram A, Opong A, Jaschke A, Hadfield J, Baschiera M, Dobson RC, Offei SK, Shepherd DN, Martin DP, Varsani A (2012) Molecular characterisation of a novel cassava associated circular ssDNA virus. Virus Res 166:130-135

9. Dayaram A, Potter KA, Pailes R, Marinov M, Rosenstein DD, Varsani A (2015) Identification of diverse circular single-stranded DNA viruses in adult dragonflies and damselflies (Insecta: Odonata) of Arizona and Oklahoma, USA. Infect Genet Evol 30:278-287

10. de Nazare Almeida Dos Reis L, Fonseca MEN, Ribeiro SG, Naito FYB, Boiteux LS, Pereira-Carvalho RC (2020) Metagenomics of neotropical single-stranded DNA viruses in tomato cultivars with and without the Ty-1 gene. Viruses 12:819

11. de Rezende RR, Mar TB, Paez LMC, Silva Xavier AD, Xavier CAD, Navas-Castillo J, Zerbini FM, Alfenas-Zerbini P (2018) Complete genome sequences of two gemycircularviruses associated with non-cultivated plants in Brazil. Arch Virol 163:3163-3166

12. Du Z, Tang Y, Zhang S, She X, Lan G, Varsani A, He Z (2014) Identification and molecular characterization of a single-stranded 
circular DNA virus with similarities to Sclerotinia sclerotiorum hypovirulence-associated DNA virus 1. Arch Virol 159:1527-1531

13. Fontenele RS, Lacorte C, Lamas NS, Schmidlin K, Varsani A, Ribeiro SG (2019) Single stranded DNA viruses associated with capybara faeces sampled in Brazil. Viruses 11:710

14. Fontenele RS, Roumagnac P, Richet C, Kraberger S, Stainton D, Aleamotu'a M, Filloux D, Bernardo P, Harkins GW, McCarthy J, Charles LS, Lamas NS, Abreu EFM, Abreu RA, Batista GB, Lacerda ALM, Salywon A, Wojciechowski MF, Majure LC, Martin DP, Ribeiro SG, Lefeuvre P, Varsani A (2020) Diverse genomoviruses representing twenty-nine species identified associated with plants. Arch Virol 165:2891-2901

15. Halary S, Duraisamy R, Fancello L, Monteil-Bouchard S, Jardot P, Biagini P, Gouriet F, Raoult D, Desnues C (2016) Novel single-stranded DNA circular viruses in pericardial fluid of patient with recurrent pericarditis. Emerg Infect Dis 22:1839-1841

16. Hanna ZR, Runckel C, Fuchs J, DeRisi JL, Mindell DP, Van Hemert C, Handel CM, Dumbacher JP (2015) Isolation of a complete circular virus genome sequence from an alaskan black-capped chickadee (Poecile atricapillus) gastrointestinal tract sample. Genome Announc 3:e01081-15

17. Hao F, Wu M, Li G (2021) Characterization of a novel genomovirus in the phytopathogenic fungus Botrytis cinerea. Virology 553:111-116

18. Jia J, Fu Y, Jiang D, Mu F, Cheng J, Lin Y, Li B, Marzano SL, Xie J (2021) Interannual dynamics, diversity and evolution of the virome in Sclerotinia sclerotiorum from a single crop field. Virus Evol 7:veab032

19. Kalyaanamoorthy S, Minh BQ, Wong TKF, von Haeseler A, Jermiin LS (2017) ModelFinder: fast model selection for accurate phylogenetic estimates. Nat Methods 14:587-589

20. Katoh K, Rozewicki J, Yamada KD (2019) MAFFT online service: multiple sequence alignment, interactive sequence choice and visualization. Brief Bioinform 20:1160-1166

21. Kazlauskas D, Dayaram A, Kraberger S, Goldstien S, Varsani A, Krupovic M (2017) Evolutionary history of ssDNA bacilladnaviruses features horizontal acquisition of the capsid gene from ssRNA nodaviruses. Virology 504:114-121

22. Kazlauskas D, Varsani A, Krupovic M (2018) Pervasive chimerism in the replication-associated proteins of uncultured singlestranded DNA viruses. Viruses 10:187

23. Kazlauskas D, Varsani A, Koonin EV, Krupovic M (2019) Multiple origins of prokaryotic and eukaryotic single-stranded DNA viruses from bacterial and archaeal plasmids. Nat Commun $10: 3425$

24. Kemenesi G, Kurucz K, Zana B, Foldes F, Urban P, Vlaschenko A, Kravchenko K, Budinski I, Szodoray-Paradi F, Bucs S, Jere C, Csosz I, Szodoray-Paradi A, Estok P, Gorfol T, Boldogh S, Jakab F (2018) Diverse replication-associated protein encoding circular DNA viruses in guano samples of Central-Eastern European bats. Arch Virol 163:671-678

25. Khalifeh A, Blumstein DT, Fontenele RS, Schmidlin K, Richet C, Kraberger S, Varsani A (2021) Diverse cressdnaviruses and an anellovirus identified in the fecal samples of yellow-bellied marmots. Virology 554:89-96

26. Kraberger S, Stainton D, Dayaram A, Zawar-Reza P, Gomez C, Harding JS, Varsani A (2013) Discovery of Sclerotinia sclerotiorum hypovirulence-associated virus-1 in urban river sediments of Heathcote and Styx Rivers in Christchurch City, New Zealand. Genome Announc 1:e00559-13

27. Kraberger S, Arguello-Astorga GR, Greenfield LG, Galilee C, Law D, Martin DP, Varsani A (2015) Characterisation of a diverse range of circular replication-associated protein encoding DNA viruses recovered from a sewage treatment oxidation pond. Infect Genet Evol 31:73-86
28. Kraberger S, Farkas K, Bernardo P, Booker C, Arguello-Astorga GR, Mesleard F, Martin DP, Roumagnac P, Varsani A (2015) Identification of novel Bromus- and Trifolium-associated circular DNA viruses. Arch Virol 160:1303-1311

29. Kraberger S, Polston JE, Capobianco HM, Alcala-Briseno RI, Fontenele RS, Varsani A (2017) Genomovirus genomes recovered from Echinothrips americanus sampled in Florida, USA. Genome Announc 5:e00445-17

30. Kraberger S, Hofstetter RW, Potter KA, Farkas K, Varsani A (2018) Genomoviruses associated with mountain and western pine beetles. Virus Res 256:17-20

31. Kraberger S, Visnovsky GA, van Toor RF, Male MF, Waits K, Fontenele RS, Varsani A (2018) Genome sequences of two single-stranded DNA viruses identified in Varroa destructor. Genome Announc 6:e00107-18

32. Kraberger S, Waits K, Ivan J, Newkirk E, VandeWoude S, Varsani A (2018) Identification of circular single-stranded DNA viruses in faecal samples of Canada lynx (Lynx canadensis), moose (Alces alces) and snowshoe hare (Lepus americanus) inhabiting the Colorado San Juan Mountains. Infect Genet Evol 64:1-8

33. Kraberger S, Cook CN, Schmidlin K, Fontenele RS, Bautista J, Smith B, Varsani A (2019) Diverse single-stranded DNA viruses associated with honey bees (Apis mellifera). Infect Genet Evol $71: 179-188$

34. Kraberger S, Schmidlin K, Fontenele RS, Walters M, Varsani A (2019) Unravelling the single-stranded DNA virome of the New Zealand blackfly. Viruses 11:532

35. Krupovic M, Ghabrial SA, Jiang D, Varsani A (2016) Genomoviridae: a new family of widespread single-stranded DNA viruses. Arch Virol 161:2633-2643

36. Krupovic M, Varsani A, Kazlauskas D, Breitbart M, Delwart E, Rosario K, Yutin N, Wolf YI, Harrach B, Zerbini FM, Dolja VV, Kuhn JH, Koonin EV (2020) Cressdnaviricota: a virus phylum unifying seven families of Rep-encoding viruses with singlestranded, circular DNA genomes. J Virol 94:e00582-20

37. Lamas NS, Fontenele RS, Melo FL, Costa AF, Varsani A, Ribeiro SG (2016) Complete genome sequence of a genomovirus associated with common bean plant leaves in Brazil. Genome Announc 4:e1247-16

38. Lamberto I, Gunst K, Muller H, Zur Hausen H, de Villiers EM (2014) Mycovirus-like DNA virus sequences from cattle serum and human brain and serum samples from multiple sclerosis patients. Genome Announc 2:e00848-14

39. Li P, Wang S, Zhang L, Qiu D, Zhou X, Guo L (2020) A tripartite ssDNA mycovirus from a plant pathogenic fungus is infectious as cloned DNA and purified virions. Sci Adv 6:eaay9634

40. Li W, Gu Y, Shen Q, Yang S, Wang X, Wan Y, Zhang W (2015) A novel gemycircularvirus from experimental rats. Virus Genes 51:302-305

41. Lima DA, Cibulski SP, Finkler F, Teixeira TF, Varela APM, Cerva C, Loiko MR, Scheffer CM, Dos Santos HF, Mayer FQ, Roehe PM (2017) Faecal virome of healthy chickens reveals a large diversity of the eukaryote viral community, including novel circular ssDNA viruses. J Gen Virol 98:690-703

42. Lima DA, Cibulski SP, Tochetto C, Varela APM, Finkler F, Teixeira TF, Loiko MR, Cerva C, Junqueira DM, Mayer FQ, Roehe PM (2019) The intestinal virome of malabsorption syndrome-affected and unaffected broilers through shotgun metagenomics. Virus Res 261:9-20

43. Liu S, Xie J, Cheng J, Li B, Chen T, Fu Y, Li G, Wang M, Jin H, Wan H, Jiang D (2016) Fungal DNA virus infects a mycophagous insect and utilizes it as a transmission vector. Proc Natl Acad Sci U S A 113:12803-12808

44. Male MF, Kami V, Kraberger S, Varsani A (2015) Genome sequences of Poaceae-associated gemycircularviruses from the Pacific Ocean island of tonga. Genome Announc 3:e01144-15 
45. Male MF, Kraberger S, Stainton D, Kami V, Varsani A (2016) Cycloviruses, gemycircularviruses and other novel replicationassociated protein encoding circular viruses in Pacific flying fox (Pteropus tonganus) faeces. Infect Genet Evol 39:279-292

46. Marzano SL, Domier LL (2016) Novel mycoviruses discovered from metatranscriptomics survey of soybean phyllosphere phytobiomes. Virus Res 213:332-342

47. Minh BQ, Schmidt HA, Chernomor O, Schrempf D, Woodhams MD, von Haeseler A, Lanfear R (2020) IQ-TREE 2: new models and efficient methods for phylogenetic inference in the genomic era. Mol Biol Evol 37:1530-1534

48. Mu F, Xie J, Cheng S, You MP, Barbetti MJ, Jia J, Wang Q, Cheng J, Fu Y, Chen T, Jiang D (2017) Virome characterization of a collection of $S$. sclerotiorum from Australia. Front Microbiol 8:2540

49. Muhire BM, Varsani A, Martin DP (2014) SDT: a virus classification tool based on pairwise sequence alignment and identity calculation. PLoS ONE 9:e108277

50. Nakasu EYT, Melo FL, Michereff-Filho M, Nagata T, Ribeiro BM, Ribeiro SG, Lacorte C, Inoue-Nagata AK (2017) Discovery of two small circular ssDNA viruses associated with the whitefly Bemisia tabaci. Arch Virol 162:2835-2838

51. Ng TF, Willner DL, Lim YW, Schmieder R, Chau B, Nilsson C, Anthony S, Ruan Y, Rohwer F, Breitbart M (2011) Broad surveys of DNA viral diversity obtained through viral metagenomics of mosquitoes. PLoS ONE 6:e20579

52. Ng TF, Chen LF, Zhou Y, Shapiro B, Stiller M, Heintzman PD, Varsani A, Kondov NO, Wong W, Deng X, Andrews TD, Moorman BJ, Meulendyk T, MacKay G, Gilbertson RL, Delwart E (2014) Preservation of viral genomes in 700-y-old caribou feces from a subarctic ice patch. Proc Natl Acad Sci U S A 111:16842-16847

53. Orton JP, Morales M, Fontenele RS, Schmidlin K, Kraberger S, Leavitt DJ, Webster TH, Wilson MA, Kusumi K, Dolby GA, Varsani A (2020) Virus discovery in desert tortoise fecal samples: novel circular single-stranded DNA viruses. Viruses 12:143

54. Pearson VM, Caudle SB, Rokyta DR (2016) Viral recombination blurs taxonomic lines: examination of single-stranded DNA viruses in a wastewater treatment plant. PeerJ 4:e2585

55. Phan TG, Mori D, Deng X, Rajindrajith S, Ranawaka U, Fan Ng TF, Bucardo-Rivera F, Orlandi P, Ahmed K, Delwart E (2015) Small circular single stranded DNA viral genomes in unexplained cases of human encephalitis, diarrhea, and in untreated sewage. Virology 482:98-104

56. Rosario K, Dayaram A, Marinov M, Ware J, Kraberger S, Stainton D, Breitbart M, Varsani A (2012) Diverse circular ssDNA viruses discovered in dragonflies (Odonata: Epiprocta). J Gen Virol 93:2668-2681

57. Rosario K, Duffy S, Breitbart M (2012) A field guide to eukaryotic circular single-stranded DNA viruses: insights gained from metagenomics. Arch Virol 157:1851-1871

58. Rosario K, Mettel KA, Benner BE, Johnson R, Scott C, YusseffVanegas SZ, Baker CCM, Cassill DL, Storer C, Varsani A, Breitbart M (2018) Virus discovery in all three major lineages of terrestrial arthropods highlights the diversity of single-stranded DNA viruses associated with invertebrates. PeerJ 6:e5761

59. Schmidlin K, Sepp T, Khalifeh A, Smith K, Fontenele RS, McGraw KJ, Varsani A (2019) Diverse genomoviruses representing eight new and one known species identified in feces and nests of house finches (Haemorhous mexicanus). Arch Virol $164: 2345-2350$

60. Siddell SG, Walker PJ, Lefkowitz EJ, Mushegian AR, Dutilh BE, Harrach B, Harrison RL, Junglen S, Knowles NJ, Kropinski AM, Krupovic M, Kuhn JH, Nibert ML, Rubino L, Sabanadzovic S, Simmonds P, Varsani A, Zerbini FM, Davison AJ (2020)
Binomial nomenclature for virus species: a consultation. Arch Virol 165:519-525

61. Sikorski A, Massaro M, Kraberger S, Young LM, Smalley D, Martin DP, Varsani A (2013) Novel myco-like DNA viruses discovered in the faecal matter of various animals. Virus Res 177:209-216

62. Siqueira JD, Curty G, Xutao D, Hofer CB, Machado ES, Seuanez HN, Soares MA, Delwart E, Soares EA (2019) Composite analysis of the virome and bacteriome of HIV/HPV co-infected women reveals proxies for immunodeficiency. Viruses 11:422

63. Smith K, Fielding R, Schiavone K, Hall KR, Reid VS, Boyea D, Smith EL, Schmidlin K, Fontenele RS, Kraberger S, Varsani A (2021) Circular DNA viruses identified in short-finned pilot whale and orca tissue samples. Virology 559:156-164

64. Somayaji V, DeNardo D, Wilson Sayres MA, Blake M, Waits K, Fontenele RS, Kraberger S, Varsani A (2018) Genome sequence of a single-stranded DNA virus identified in Gila monster feces. Microbiol Resour Announc 7:e00925-e1018

65. Steel O, Kraberger S, Sikorski A, Young LM, Catchpole RJ, Stevens AJ, Ladley JJ, Coray DS, Stainton D, Dayaram A, Julian L, van Bysterveldt K, Varsani A (2016) Circular replication-associated protein encoding DNA viruses identified in the faecal matter of various animals in New Zealand. Infect Genet Evol 43:151-164

66. Thi Kha TuN, Thi Thu Hong N, Thi Han Ny N, My Phuc T, Thi Thanh Tam P, Doorn HRV, Dang Trung Nghia H, Thao Huong D, An Han D, Thi Thu Ha L, Deng X, Thwaites G, Delwart E, Virtala AK, Vapalahti O, Baker S, Van Tan L (2020) The virome of acute respiratory diseases in individuals at risk of zoonotic infections. Viruses 12:960

67. Tisza MJ, Pastrana DV, Welch NL, Stewart B, Peretti A, Starrett GJ, Pang YS, Krishnamurthy SR, Pesavento PA, McDermott DH, Murphy PM, Whited JL, Miller B, Brenchley J, Rosshart SP, Rehermann B, Doorbar J, Ta'ala BA, Pletnikova O, Troncoso JC, Resnick SM, Bolduc B, Sullivan MB, Varsani A, Segall AM, Buck CB (2020) Discovery of several thousand highly diverse circular DNA viruses. Elife 9:e51971

68. Uch R, Fournier PE, Robert C, Blanc-Tailleur C, Galicher V, Barre R, Jordier F, de Micco P, Raoult D, Biagini P (2015) Divergent gemycircularvirus in HIV-positive blood, France. Emerg Infect Dis 21:2096-2098

69. van den Brand JM, van Leeuwen M, Schapendonk CM, Simon JH, Haagmans BL, Osterhaus AD, Smits SL (2012) Metagenomic analysis of the viral flora of pine marten and European badger feces. J Virol 86:2360-2365

70. Varsani A, Krupovic M (2017) Sequence-based taxonomic framework for the classification of uncultured single-stranded DNA viruses of the family Genomoviridae. Virus Evol 3:vew037

71. Waits K, Edwards MJ, Cobb IN, Fontenele RS, Varsani A (2018) Identification of an anellovirus and genomoviruses in ixodid ticks. Virus Genes 54:155-159

72. Walker PJ, Siddell SG, Lefkowitz EJ, Mushegian AR, Adriaenssens EM, Dempsey DM, Dutilh BE, Harrach B, Harrison RL, Hendrickson RC, Junglen S, Knowles NJ, Kropinski AM, Krupovic M, Kuhn JH, Nibert M, Orton RJ, Rubino L, Sabanadzovic S, Simmonds P, Smith DB, Varsani A, Zerbini FM, Davison AJ (2020) Changes to virus taxonomy and the Statutes ratified by the International Committee on Taxonomy of Viruses (2020). Arch Virol 165:2737-2748

73. Wang H, Li S, Mahmood A, Yang S, Wang X, Shen Q, Shan T, Deng X, Li J, Hua X, Cui L, Delwart E, Zhang W (2018) Plasma virome of cattle from forest region revealed diverse small circular ssDNA viral genomes. Virol J 15:11

74. Wang J, Li Y, He X, Ma J, Hong W, Hu F, Zhao L, Li Q, Zhang J, Zhang C, Zhang F (2019) Gemykibivirus genome in lower respiratory tract of elderly woman with unexplained acute respiratory distress syndrome. Clin Infect Dis 69:861-864 
75. Wang Y, Yang S, Liu D, Zhou C, Li W, Lin Y, Wang X, Shen Q, Wang H, Li C, Zong M, Ding Y, Song Q, Deng X, Qi D, Zhang W, Delwart E (2019) The fecal virome of red-crowned cranes. Arch Virol 164:3-16

76. Weber MN, Cibulski SP, Olegario JC, da Silva MS, Puhl DE, Mosena ACS, Alves C, Paim WP, Baumbach LF, Mayer FQ, Fernandes ARF, Azevedo SS, Canal CW (2018) Characterization of dog serum virome from Northeastern Brazil. Virology 525:192-199

77. Wu Z, Yang L, Ren X, He G, Zhang J, Yang J, Qian Z, Dong J, Sun L, Zhu Y, Du J, Yang F, Zhang S, Jin Q (2016) Deciphering the bat virome catalog to better understand the ecological diversity of bat viruses and the bat origin of emerging infectious diseases. ISME J 10:609-620

78. Yang F, Yang X, Wu K (2020) A novel circular Rep-encoding single-stranded DNA virus detected in Agrotis ipsilon (Lepidoptera: Noctuidae) in China. Arch Virol 165:771-774

79. Yu X, Li B, Fu Y, Jiang D, Ghabrial SA, Li G, Peng Y, Xie J, Cheng J, Huang J, Yi X (2010) A geminivirus-related DNA mycovirus that confers hypovirulence to a plant pathogenic fungus. Proc Natl Acad Sci U S A 107:8387-8392
80. Zhang W, Li L, Deng X, Blumel J, Nubling CM, Hunfeld A, Baylis SA, Delwart E (2016) Viral nucleic acids in human plasma pools. Transfusion 56:2248-2255

81. Zhang W, Yang S, Shan T, Hou R, Liu Z, Li W, Guo L, Wang Y, Chen P, Wang X, Feng F, Wang H, Chen C, Shen Q, Zhou C, Hua X, Cui L, Deng X, Zhang Z, Qi D, Delwart E (2017) Virome comparisons in wild-diseased and healthy captive giant pandas. Microbiome 5:90

82. Zhao L, Rosario K, Breitbart M, Duffy S (2019) Eukaryotic circular Rep-encoding single-stranded DNA (CRESS DNA) viruses: ubiquitous viruses with small genomes and a diverse host range. Adv Virus Res 103:71-133

83. Zhou C, Zhang S, Gong Q, Hao A (2015) A novel gemycircularvirus in an unexplained case of child encephalitis. Virol J 12:197

Publisher's Note Springer Nature remains neutral with regard to jurisdictional claims in published maps and institutional affiliations. 